\title{
POLITIQUE, TÉLÉVISION ET SPECTACLE : LE CAS DE LA FLANDRE
}

\author{
Daniel Biltereyst et Lieve Desmet ${ }^{1}$
}

\begin{abstract}
Network news oscillates vividly between sobriety and spectacle. It upholds the tradition of informed citizenship in its structure of credible anchors and diligent reporters, objective reporting styles, canons of validation for evidence, and priority for issues pertaining to the nation-state.
\end{abstract}

Bill Nichols (1994: 48-49)

L'histoire de la télévision flamande, qui a débuté en 1953, s'apparente étroitement à un récit politique. De nombreuses études ont montré en détail l'influence exercée par les partis sur les aspects les plus divers de ce média (voir e.a. Burgelman, 1990). En Flandre, les trois grandes familles traditionnelles - libéraux, socialistes et démocrates chrétiens ont tenté d'influencer l'audiovisuel dans toutes ses composantes. Cette confusion d'intérêts a pris des formes variées, allant de l'ingérence dans la gestion du personnel (techniciens, journalistes ou fonctions dirigeantes) à des tentatives obstinées pour contrôler l'information. Le travail de la rédaction a été attentivement supervisé par les différents quartiers généraux des partis. Le journal télévisé et l'information poli-

1 Daniel Biltereyst est professeur et Lieve Desmet chercheuse au Département des Sciences de la Communcation à l'Université de Gand.

Recherches en communication, $\mathrm{n}^{\circ} 24$ (2005). 
tique, en particulier, ont toujours constitué la pierre d'angle de leur stratégie.

La relation entre classe politique et information politique télévisée a donc toujours représenté un sujet brûlant pour l'opinion publique. Outre la recherche académique, (voir e.a. Burgelman, 1990; Witte, 2002; Desmet, 2005), l'information politique à la télévision a fait l'objet de débats plus larges. Une critique souvent exprimée dans ce cadre consiste à dire que, sous la pression de la concurrence et de la commercialisation, la télévision a de plus en plus manqué à son devoir démocratique fondamental, à savoir tenir lieu de forum public accessible pour une information indépendante et critique (Witte, 2002). D'autres ont prétendu que les règles traditionnelles de la télévision, notamment sa tendance à la dramatisation, à la superficialité et à la mise en évidence des conflits, avaient eu une influence non négligeable sur l'entreprise politique elle-même. Cette critique sur l'impact néfaste du média sur la démocratie s'est traduite dans le débat public flamand par des expressions comme dramadémocratie (Elchardus, 2002).

Dans une «dramadémocratie », les hommes politiques doivent dans une large mesure tenir compte des médias, en particulier des règles de la télévision. La perception devient plus importante que le contenu. Une citation efficace vaut plus qu'une analyse bien étayée. Les partis s'entourent de script doctors et de marketeers, dont le but est d'accompagner les hommes politiques dans un espace public qui est largement déterminé par la télévision et les règles qui y sont en vigueur. Un politicien à l'allure jeune et sympathique, qui s'exprime bien et lâche de temps à autre un scoop, voit sa valeur politique (c'est-à-dire médiatique) augmenter. En Flandre, disent les critiques, cette interaction entre le politique et le monde des médias est intense. Cela se voit à la manière dont les partis engagent systématiquement des bekende Vlamingen, c'est-à-dire des célébrités du petit écran ou du show-business, qu'ils placent bien haut sur leur liste électorale. Les candidats jeunes et séduisants reçoivent la priorité sur les hommes de dossiers. Les hommes politiques ne ratent pas une occasion d'apparaittre dans les programmes de jeux et autres. Dans un tel contexte, le risque de voir les médias s'insinuer de plus en plus dans leur sphère privée augmente en proportion. De plus, les médias et la télévision en particulier doivent affronter une concurrence et une commercialisation de plus en plus féroces, ce qui peut aussi influencer la forme et le contenu de l'information. Les motsclés sont, dans ce domaine, l'influence croissante de la dramatisation, la mise en exergue des conflits, la spectacularisation et la personnali- 
sation. Dès lors que l'information obéit de plus en plus aux règles du divertissement, elle glisse dans la direction de ce que les Anglo-saxons nomment infotainment.

Ces développements semblent à tout le moins modifier en profondeur la distribution traditionnelle des rôles entre médias et politique. La question est de savoir si cette critique est fondée. Et surtout, si toutes ces tendances sont réellement neuves. Dans cet exposé, nous nous pencherons sur les débats politiques télévisés en Flandre. Après une brève analyse du débat public et académique critique sur l'information politique en Flandre, nous dresserons un historique succinct des programmes politiques de la chaîne publique flamande en insistant particulièrement sur les changements de format qu'ils ont subis.

\section{Infotainment, dramadémocratie, spectacle médiatique}

Au moment même où nous rédigeons ce texte, un débat passionné agite une fois de plus la Flandre sur la façon donc les hommes politiques sont projetés par les médias dans l'espace public. Dans un petit article, le journal Le Soir (10/01/06) a annoncé que Rik Daems, chef des libéraux flamands (VLD) à la Chambre fédérale des représentants, entretenait une liaison avec une collègue du parti socialiste francophone (PS). Ce n'était pas la première fois que l'homme faisait parler de lui. Le moulin à rumeurs médiatique avait déjà évoqué à plusieurs reprises ses problèmes conjugaux et ses péripéties sentimentales. Mais cette fois, Daems ne se contentait pas de franchir la frontière linguistique, il choisissait sa compagne dans une famille politique contre laquelle il s'était auparavant battu bec et ongles. Le Soir signalait aussi que la députée en question, Sophie Pécriaux, était enceinte. À la même période, Daems avait encore accordé une interview croustillante à Dag allemaal (10/01/06), un hebdomadaire de programmes TV aux allures de tabloïd un peu rance, dans lequel il avait évoqué ses problèmes conjugaux, mais sans dire mot ni de cette relation extraconjugale, ni de la grossesse. Lorsque les commentateurs, qui suivent la politique intérieure de près, ont également appris que le Premier ministre Guy Verhofstadt, lui-même membre du VLD, cherchait ouvertement un nouveau boulot pour Daems, la digue a cédé. Les problèmes de Daems se sont soudain transformés en affaire publique. Dans une réaction de crise, Daems a annoncé, probablement sous la pression de la direction du VLD, qu'il renonçait à ses fonctions de chef de groupe. Qui pouvait 
encore le prendre au sérieux dans ses attaques contre les socialistes francophones, à présent qu'il partageait ses nuits avec un membre féminin, connu, de ce parti ?

Ce fait d'actualité, qui a disparu de la une au bout d'une semaine, illustre une fois encore la relation délicate existant entre médias et monde politique, surtout en ce qui concerne la ligne de démarcation entre sphère publique et sphère privée. Dans quelle mesure les médias pouvaient-ils dévoiler la liaison de Daems avec Pécriaux ? La relation amoureuse de Daems avec une femme politique de l'autre groupe linguistique et d'un parti que Daems avait toujours vivement critiqué avait-elle ou non une incidence sur ses activités de chef de groupe libéral au Parlement? Les médias devaient-ils en parler, alors que la démission de Daems lui était en partie dictée par la direction de son parti ? Cette affaire privée n'était-elle pas par essence politique ? Ces questions et bien d'autres, notamment celle du rôle des médias, ont été abondamment évoquées dans les articles d'opinion, les éditoriaux, les débats et autres forums. Les programmes télévisés, des journaux parlés aux magazines d'information en passant par les talk-shows, ont eux aussi largement commenté l'affaire.

Les éditorialistes et les journalistes n'ont pas été les seuls à intervenir dans le débat. Des hommes politiques, des universitaires et d'autres faiseurs d'opinion ont également critiqué le rôle des médias. Les journaux télévisés, les magazines et les programmes d'information ont été particulièrement visés. D'une manière générale, le débat était dominé par une vision classique du rôle démocratique des médias, selon laquelle l'information et ses interprétations constituent des forums centraux pour la diffusion et la «consommation » d'une information sociale pertinente. Dans cette vision, les médias informent le citoyen d'une façon objective, indépendante et noble.

Dans le débat public autour de l'affaire Daems, il a plus d'une fois été fait allusion à la crainte de voir les médias de qualité et la télévision publique manier eux aussi des techniques d'entertainment commercial dans leur mission d'information. Cela peut aller de l'exploitation de la sphère privée de personnages publics à l'emploi de techniques de dramatisation en passant par le sensationnalisme. Cette inquiétude ne concerne pas seulement les journaux, qui se battent en Flandre comme en Belgique francophone contre une diminution de leur lectorat et une concurrence croissante. Les chaînes de télévision, y compris la télévision publique, sont également engagées dans une lutte de concurrence intense, dans laquelle la conquête du public et la rationalisation sont 
devenues des thèmes centraux. Avec le risque que, dans notre dramadémocratie, la télévision ne s'oriente de plus en plus vers l'infotainment.

En Flandre comme ailleurs (Hooghe e.a., 2005), aucune catégorie de programmes n'a bénéficié, ces derniers temps, d'autant d'attention que les actualités et les programmes d'information politique. Il est vrai qu'ils ont subi des transformations radicales, surtout au niveau de la forme. Au cours des quinze dernières années, l'offre d'information est assurément devenue bien plus dynamique et cinétique (changements d'images et mouvements de caméra plus rapides). Le son et la musique y ont conquis une place importante et le rôle du présentateur a changé (personnalisation par le biais du présentateur vedette). Cette méthode et d'autres ont été vivement stigmatisées par la critique, qui les a intégrées dans un discours sur l'entertainment. La manière dont l'actualité est présentée au citoyen est capitale. Dans les journaux télévisés traditionnels, l'accessibilité avait certes son importance, mais dans la pratique, elle laissait parfois à désirer ; toutes les études sur la compréhension et l'accessibilité montrent que les gens avaient de sérieuses difficultés avec ce type de programmes d'information «dure». Cela tenait non seulement à l'enveloppe technique et formelle, mais aussi et surtout au langage utilisé et au ton (mode of address). Celui-ci était jugé trop abstrait, trop distant et trop difficile. La transformation de la forme des journaux télévisés n'a donc pas seulement été motivée par la tendance à la commercialisation, mais aussi par un souci d'accessibilité et de compréhension. Aujourd'hui, l'offre d'information se caractérise, davantage que dans le passé, par l'emploi de techniques qui relèvent plutôt de la fiction et de l'entertainment (personnalisation, dramatisation, accentuation des conflits). Elle n'échappe pas non plus à l'influence du phénomène du first person media. Par cette expression, Dovey (2000: 1) désigne la tendance croissante des médias à mettre l'accent sur les modes d'expression subjectifs, autobiographiques et intimes. Dovey observe que l'attention pour les expériences subjectives individuelles grandit non seulement dans la téléréalité, mais aussi dans les informations. Cette tendance se vérifie dans une offre d'actualités centrées sur les intérêts et la sphère d'intérêt du citoyen (façon plus directe d'aborder le public, techniques de bottom-up, langage simple).

D'un point de vue critique, tous ces changements formels sont généralement considérés comme symptomatiques d'un glissement vers l'infotainment et la commercialisation. Les recherches menées sur la forme des actualités ne confirment toutefois pas entièrement ces hypothèses et font état, au-delà des facteurs de production technologiques 
et techniques, d'une évolution mixte. D'une part, on constate l'emploi d'une langue plus simple, plus accessible, et de jugements plus subjectifs dans les nouvelles. De l'autre, les rédactions des journaux télévisés semblent, en Europe occidentale du moins, opter pour le maintien d'un équilibre avec une information plus dure et une analyse plus abstraite. C'était aussi la position de Klaus Van Isacker (Information VTM), qui ajoutait que l'émetteur commercial veillait à « limiter les interviews de rues et à ne jamais les diffuser en dehors d'un contexte d'information dure ». En d'autres mots, les actualités manient un discours mixte. Audelà du journal télévisé, la critique relative à l'infotainment s'adresse surtout aux magazines d'actualité « grand public » diffusés en marge de celui-ci (par exemple, Koppen sur la chaîne publique flamande VRT). C'est principalement dans ce type de programmes que les techniques de dramatisation, de sensationnalisation et de personnalisation sont les plus manifestes: on attache plus d'importance à la personne qui se cache derrière l'homme politique qu'à son projet social. Mais les créateurs de programmes évacuent ces critiques en invoquant le besoin d'accessibilité et, même, un certain projet de démocratisation. Cette argumentation touche d'ailleurs au cœur même du débat sur l'importance sociale des journaux télévisés et des magazines d'information. D'une part, les raisons de la transformation ne sont pas toujours claires : s'agit-il seulement de commercialisation, ou le souci d'une plus grande accessibilité intervient-il ? D'autre part, il faut s'assurer que la question est réellement pertinente. Dans le prolongement direct de cette vision sur l'ouverture relative de la consumer culture, on peut se demander si les développements évoqués n'offrent effectivement pas aux idéaux du service public certaines opportunités de démocratisation, comme l'élargissement du débat public, l'accessibilité et la participation. Vus sous cet angle, les nouveaux développements des journaux télévisés et des programmes d'information semblent positifs. Mais une condition supplémentaire cruciale concerne la valeur de leur contenu, autrement dit, la valeur de l'information en termes de qualité, de diversité et d'indépendance.

\section{Les débats politiques}

La longue tradition d'immixtion politique dans le fonctionnement de la télévision flamande ne s'est pourtant pas manifestée d'emblée par une présence importante des hommes politiques dans les actualités elles-mêmes. Les politiciens, des ministres aux bourgmestres, occu- 
paient surtout l'écran à l'occasion des cérémonies officielles, des fêtes populaires et des inaugurations (Desmet, 2005:159). Contrairement à ce que l'on pense généralement, ils n'apparaissaient pas forcément, à l'époque, dans des magazines d'information sérieux. Beaucoup tentaient plutôt d'accroître leur notoriété et leur popularité en participant à des programmes de jeux destinés au grand public. Pendant les cinq premières années de la télévision flamande, le centre de gravité de l'information et de l'actualité politiques est resté dans la presse écrite et la radio. Les sujets de politique intérieure ou les interviews d'hommes politiques passaient rarement à l'écran. L'actualité politique était réservée au « journal parlé », une partie du journal télévisé pendant laquelle les sujets étaient présentés de façon quasiment radiophonique.

Un revirement concernant la présence d'hommes politiques à la télévision flamande s'est produit lors de l'Exposition universelle de 1958, à Bruxelles. À cette occasion, la télévision a considérablement augmenté ses temps d'émission. Pour les hommes politiques, c'était une chance unique de se mettre sous le feu des projecteurs, d'autant que la télévision était elle-même en quête d'interlocuteurs et de lieux d'enregistrement ${ }^{1}$. Dans la même période, les hommes politiques ont également commencé à apparaître de plus en plus fréquemment dans les jeux télévisés. Au début des années soixante en particulier, nos dirigeants ont commencé à apparaître dans des programmes comme Een tegen allen (1963) et Harten drie (1964) (De Volksgazet, 4-5 juillet 1964).

L'offre de programmes politiques «sérieux » s'est également étoffée, avec pour objectif de rendre compte des thèmes et des événements du moment. Un développement important dans ce cadre a été induit par la loi de 1960 sur la radiotélévision. Cette loi impartissait notamment un temps d'antenne aux partis politiques, qui ont alors assuré des Gastprogramma's, rebaptisés plus tard Programma's door derden. Par ailleurs, la BRT (nouvelle appellation de la NIR) présentait régulièrement des «Communications gouvernementales ». Fournies par le gouvernement, ces séquences ne relevaient pas non plus, ni pour leur contenu ni pour leur forme, de la rédaction de la télévision publique.

Si nous dressons la liste des émissions politiques dont la télévision flamande était responsable du point de vue rédactionnel, nous obtenons inévitablement un large éventail de types de programmes et de formats. À côté du Journaal et des débats comme Panorama (1953-), Terzake,

1 VRT-Documentenarchief, Sectie Varia, Dossier 1956 - 1957. 
Koppen et Zinzen (2002), les actualités politiques ont conquis une place centrale dans la mission d'information de la télévision publique.

Dès le début, la télévision a présenté quelques émissions d'information dure, lors desquelles les principaux acteurs politiques s'opposaient dans des débats et présentaient leurs points de vue au public. On peut citer comme exemple Ieder zijn waarheid (1953-1962), un débat mensuel qui réunissait des représentants des trois grands partis. Sous la direction d'un modérateur (par ex. Nic Bal, Karel Goris et Karel Hemmerechts), d'importants sujets étaient débattus. L'argumentation rationnelle et la connaissance des dossiers primaient, même si la dramatisation et la personnalisation n'étaient jamais bien loin. Dans les années soixante, ce magazine de discussion a été remplacé par Debatten en gesprekken (1967-1973) et Standpunten (1969-1973), qui utilisaient plus ou moins la même formule. En conséquence de la montée et du succès électoral de la Volksunie (parti nationaliste flamand), ces débats accueillaient désormais quatre partis, souvent représentés par leur président.

Dans les années septante, l'offre de ce type de programmes de discussion pure et dure a été élargie. À partir de 1973, le service d'information a fusionné Ieder zijn waarheid et Debatten en gesprekken en une nouvelle production hebdomadaire. Confrontatie (1973-1988) a d'abord été diffusé tous les mercredis soirs, puis, à partir de 1976, le dimanche matin. Ce programme correspondait également au concept traditionnel des actualités dans lequel les hommes politiques parlent en tant qu'experts, de façon rationnelle, sur des sujets publics (public matters). Souvent, Confrontatie abordait un seul thème d'actualité politique, qui était traité en profondeur sous la direction d'un modérateur bien informé. Ce dernier n'était toutefois pas seulement un expert : il jouait aussi un rôle de meneur de jeu. En raison de la règle d'objectivité en vigueur, il devait veiller soigneusement à ce que les participants reçoivent tous plus ou moins le même temps de parole. Ce format sévère, distant, se traduisait formellement dans une mise en scène rigide sans beaucoup de fioritures. Pour ces programmes de studio, on faisait usage d'une multicaméra statique et d'un décor monotone qui ne risquait pas de distraire l'attention du spectateur. Conformément à une conception paternaliste et distanciée de la mission d'information, le contenu et l'argumentation de la discussion publique étaient prioritaires.

En 1988, Confrontatie a été remplacé par De zevende dag, un nouveau talk-show conçu comme une séance de bavardage dans un café, le dimanche matin. L'apparence sévère de l'émission a donc cédé 
la place à un format plus dynamique. On a cessé de ne commenter qu'un seul sujet. De zevende dag comportait les rubriques fixes suivantes : une accroche (retour sur une image, un fait, une personnalité de la semaine), «zeven op zeven » (un résumé des événements de la semaine) et «confrontatie » (un débat). Désormais, les thèmes d'actualité étaient davantage abordés «entre la poire et le fromage », tandis que les hommes politiques étaient de plus en plus concurrencés par des citoyens ordinaires qui prenaient la parole. Le décor sévère, jugé ennuyeux, a pris de la profondeur. Mais le changement le plus marquant a consisté à inviter un public, qui suivait (plus ou moins attentivement) les conversations à table. De zevende dag illustrait ainsi l'idée d'un marché ou d'un forum, avec une assistance à l'arrière-plan. Le public incarnait l'illusion d'un citoyen participatif. Le nouveau format impliquait aussi un maniement plus souple de la caméra. Une place importante était réservée à la musique, censée souligner l'alternance d'entretiens et de sujets. Le débat politique se rapprochait ainsi des talkshows plus populaires. Le style d'animation rigide de Confrontatie a également été adapté : plusieurs présentateurs intervenaient désormais en alternance et la musique jouait un rôle de premier plan.

Ces transformations fondamentales doivent naturellement être replacées dans un cadre plus large. La télévision publique était à l'époque talonnée par la télévision commerciale (Vlaamse Televisie Maatschappij), créée en février 1989. Menacée dans son monopole, elle a entamé, à partir de 1988, une réforme en profondeur. Celle-ci comprenait notamment un renouvellement radical de la forme et du contenu d'émissions comme Confrontatie, considérées comme trop rigides et trop distantes. Par son format, De zevende dag s'inscrivait tout à fait dans la ligne d'une approche plus douce, participative. L'information dure sur les sujets politiques du moment gardait néanmoins son importance. La discussion entre experts et hommes politiques était maintenue pour une part. Mais le concept traditionnel était désormais complété par une attention accrue pour des sujets d'intérêt public et des thèmes sociaux plus sensibles. Le spectateur était plus étroitement impliqué. Le ton devenait plus dynamique et plus direct.

Des modifications plus nettes encore ont suivi. Une importante adaptation a eu lieu à l'occasion du projet de réforme « Nieuwsproject 2002 », dirigé par Leo Hellemans. L'interactivité avec le public est devenue centrale et davantage de sujets ont été abordés. Il était rare qu'un thème soit traité pendant plus de vingt minutes. La mise en scène sévère de la partie «confrontatie » a totalement disparu au profit 
d'un bavardage léger dans des sièges confortables. L'atmosphère était inspirée par l'idée, très en vogue, du cocooning.

\section{Émissions électorales et autres émissions politiques}

Ces transformations sont également perceptibles dans d'autres sortes de programmes proposés par le service information. Une analyse similaire peut être faite sur les émissions électorales, qui ont peu à peu pris l'allure de show spectaculaires et grandioses. Ces évolutions dans la forme et le contenu des programmes d'information offrent pourtant un contraste criant avec le discours public et la politique de base adoptée par la télévision publique flamande au cours de ses cinquante ans d'existence. Le service public a toujours cherché à offrir un dosage politiquement correct. En raison de cette représentation équitable des partis, la politique de programmation politico-culturelle dans son ensemble a dû et doit encore respecter une répartition précise de son temps d'antenne (Ceuleers, 1980 ; Desmet, 2005 ; Witte, 2002). Souvent, cela s'est traduit par un contrôle presque anxieux des temps de parole accordés aux représentants des principaux partis.

La situation a progressivement évolué. Avant les années quatrevingt, les nouvelles formations politiques menaient des actions de protestation pour obtenir une représentation. Ces campagnes, parfois audacieuses, créaient la sensation. Comme exemple symbolique, citons l'action lors de laquelle les membres de la Volksunie ont envahi les studios pendant la diffusion du jeu Wie weet wat pour brandir des pamphlets devant les caméras (Anthierens, 1963). Ce faisant, les partis créaient un spectacle télévisé au sens littéral! Le mouvement s'est inversé à partir des années quatre-vingt, époque à laquelle la tendance sociale de plus en plus nette qui consistait à prêter davantage intérêt aux minorités a commencé à imprégner la mentalité des programmateurs. La télévision a elle-même pris l'initiative d'offrir un forum aux petits partis récemment créés (souvenons-nous par exemple de l'attention accordée au parti ROSSEM au début des années nonante). Ce mouvement a engendré et alimenté un débat sur le rôle de la télévision dans l'émergence des partis politiques.

Les changements concernant le format, la mise en scène, la présentation et la participation ont suivi la tendance générale des programmes d'information. Hartley (2002: 119) a décrit cette évolution comme le passage d'un média «parlant» (talking) et «vidéo » à un média « hybridant» (hybridising) et «poreux» (porous). Le fait que, pour 
les émissions politiques en tout cas, chacun de ces changements ait été perçu à son époque comme une augmentation du caractère spectaculaire, constitue en soi une observation importante. Cela révèle-til des difficultés générales d'adaptation réciproque, une inertie de la part du politique et du pouvoir, une popularisation et un nivellement de la politique par les concepteurs médiatiques ou des incompatibilités fondamentales ? Autant de questions intrigantes, auxquelles il n'est pas possible de donner une réponse claire dans le cadre de cet exposé.

Ce qui est clair en revanche, c'est que les programmes politiques en studio sont de plus en plus souvent mis en scène, et ce, en fonction, semble-t-il, des règles propres au divertissement (Desmet, 2005). Dans les années cinquante, on «débattait » dans des programmes comme Ieder zijn waarheid, sous l'œil attentif d'un journaliste, chacun à son tour et avec un même temps de parole. La politique étrangère, unique sujet à l'origine, a dès la première année été élargie à des sujets nationaux qui « touchaient le spectateur de plus près ${ }^{1} »$. En revanche, il a fallu attendre 1973 pour qu'intervienne une première transformation importante du débat en studio. La nouvelle émission, Confrontatie, conservait la formule de base, mais y ajoutait quelques reportages. Depuis le passage à De zevende dag en 1988, on a privilégié des techniques qui renforçaient de plus en plus et de façon plus manifeste le caractère spectaculaire. Citons à cet égard l'emploi de caméras mobiles, les nombreux changements de prises de vues, l'emploi du zoom, la présence d'un public participatif dans le studio, les interpellations téléphoniques de spectateurs, etc. $^{2}$

Cette évolution s'est également étendue aux émissions électorales. Dans les premières années de la télévision, le service d'information organisait souvent un seul débat, qui réunissait non pas des hommes politiques, mais des journalistes de la presse écrite représentant les différents groupes politiques. Ce format ne rencontrait toutefois guère de succès. À partir de 1968, la BRT a organisé une émission le jour même des élections. Contrairement à ses collègues de la RTB, qui en faisaient une «Election Party», la BRT a d'abord opté pour une présentation nettement plus sobre des résultats, agrémentée de quelques variétés et des interventions des professeurs Demeyer (KUL) et Picard (RUG) (Humo, 28 mars 1968). En 1974, à l'époque où Ceuleers était

1 VRT-Documentenarchief, Sectie Informatie, Activiteitsverslag van de sectie informatie (1/4/54 - 31/12/54).

2 http://users.pandora.be/vrtnieuws/geschiedenisdezevendedag.htm, novembre 2001. 
rédacteur, on a lancé un show électoral plus large. La formule, soutenue par des techniques de plus en plus ingénieuses, s'est maintenue jusqu'à aujourd'hui. Le concept des débats « monstres » n'a été développé qu'à la fin des années septante. Dans ce cadre, cinq représentants des différents partis devaient à chaque fois répondre à une vingtaine d'opposants. Un journaliste tenait lieu de présentateur et de modérateur. En 1980, Jan Ceuleers (1980: 25) se demanda si le succès de ce genre de débats n'était pas dû au facteur spectaculaire bien plus qu'au contenu.

Un gros changement s'est produit en 2000, lorsque la télévision a franchi un pas de plus en créant l'émission Bracke \& Crabbé. Cette série avait toutes les caractéristiques d'un show quotidien, à la mise en scène fluide. Précédant les élections elles-mêmes, Bracke \& Crabbé introduisait une formule amusante d'entretiens avec de jeunes hommes politiques confirmés ou montants, combinés à des jeux, à des sujets ludiques et à toutes sortes de reportages. L'émission n'a pas tardé à susciter de nombreux débats, lors desquels ses détracteurs ont violemment fustigé les éléments «show » et «jeux» du programme. Un traitement aussi léger de la politique et des élections défie l'imagination, a t-on entendu dire. Mais le débat n'a pris de l'intérêt que lorsque l'émission a trouvé des défenseurs. Un solide argument en faveur de Bracke \& Crabbé était que le programme offrait un meilleur éclairage à l'activité politique et la rendait plus accessible. Le succès de ce type de programmes semblait le confirmer, puisque même des gens qui regardaient rarement les nouvelles comptaient parmi les spectateurs du nouveau talk-show politique. Siegfried Bracke, l'un des présentateurs, s'est posé la question de savoir si la télévision publique ne devait pas, dans un projet global de démocratisation, travailler pour les gens qui comprennent, possèdent et savent moins, afin d' « ouvrir par ce biais la voie à davantage de démocratie » (voir Biltereyst, 2000).

Le reportage parlementaire (en direct), autre format de programme, a toujours constitué un point délicat (pour un aperçu, voir Witte et Ceuleers, 2003). Bien que ce type d'informations soit souvent inclus dans le Journaal et dans les magazines, des tentatives ont également été entreprises pour créer des émissions spécialisées à partir des débats du Parlement. Les essais de téléréalité depuis la Chambre (1977-1979) ont tourné court (Witte et Ceuleers, 2003:332). Exception faite du reportage spécial concernant les activités de la commission d'enquête parlementaire « Dutroux » (1997), il a fallu attendre 2002 pour qu'un magazine politique, Villa Politica, soit entièrement consacré à l'information parlementaire. 


\section{Conclusion}

Une critique importante, souvent entendue dans les débats récents concernant l'actualité et l'information politique à la télévision, porte sur le fait que ces catégories n'ont pas pu se soustraire à une transformation plus vaste de la télévision, se traduisant par les phénomènes suivants : attention grandissante pour le sensationnel et les nouvelles d'intérêt général (et marginalisation parallèle des nouvelles politiques), emploi plus large de techniques d'infotainment (personnalisation, dramatisation,...), plus d'information douce, intérêt accru pour la perception des faits d'actualité par le consommateur-citoyen (microstrottoirs, désaffection à l'égard des experts), plus de nouvelles nationales au détriment de l'information internationale, etc. En outre, une certaine standardisation aurait eu lieu dans les actualités télévisées. Les spécialistes inscrivent ces changements dans un cadre plus large, dans lequel la «tabloïdisation », l'infotainment ou l'essor de la téléréalité et de la tendance first person media ont également leur place (Dovey, 2000). Ces développements funestes sont souvent associés à la crainte d'une diminution de la participation sociale, considérée comme un indicateur du désintérêt croissant porté à la politique et à la démocratie. Aux États-Unis, un sondage d'opinion annuel a montré que la consommation d'actualités via les mass media était en diminution. Seuls les groupes plus instruits et plus âgés manifestent un intérêt durable pour les nouvelles; les journaux télévisés nationaux et les quotidiens ont de plus en plus de mal à survivre. Selon le même sondage, le public américain s'intéresse surtout aux crimes, aux nouvelles locales et à la santé, tandis que la culture, l'art et surtout les nouvelles internationales figurent tout en bas de la liste. Poussés par la concurrence, les chiffres de tirage et d'audimat, les médias d'information semblent vouloir donner au public ce qu'il attend : moins de nouvelles étrangères, des informations plus accessibles, plus sensationnelles, centrées sur des faits locaux... Les nouvelles se réduisent peu à peu à un simple produit commercial. On ne peut pas encore affirmer avec certitude que ce soit le cas en Flandre, mais il est indéniable que, chez nous aussi, certaines des techniques de dramatisation, de spectacularisation et d'infotainment décrites ci-dessus pèsent sur l'information politique. 
